doi:10.17659/01.2017.0093

Journal of Case Reports 2017;7(4):341-344

\title{
Primary Central Nervous System B Cell Lymphoma, Unclassifiable, in an Immunocompetent Young Male
}

\author{
Meera PP, Bhulaxmi $\mathrm{P}^{1}$ \\ Departments of Pathology and ${ }^{1}$ Laboratory Medicine, Yashoda Hospital, Somajiguda, Hyderabad, Telangana-500082, India.
}

\section{Corresponding Author:}

Dr. Meera PP

Email: meerapoonacha@gmail.com

This is an Open Access article distributed under the terms of the Creative Commons Attribution License (creativecommons.org/ licenses/by/3.0).

Received Accepted

Published
July 28, 2017

October 1, 2017

October 20, 2017

\begin{abstract}
Background: Primary CNS B cell lymphoma, unclassifiable with features intermediate between diffuse large B cell lymphoma and Burkitt's lymphoma (DLBCL/BL) in an immuno-competent young boy is a rare entity. Case Report: A 14 year old boy presented with headache and vomiting. CT brain showed well-defined mildly enhancing hyperdense lesion in left intra-ventricular region with seeding in the temporal lobe and the supra-sellar region suggesting a neoplastic condition. Squash cyotology of specimen after tumor decompression suggested high grade non-Hodgkin's lymphoma. Tissue paraffin sections showed tumor cells diffusely positive for $\mathrm{CD} 20, \mathrm{BCl} 6$, and $\mathrm{BCl} 2$, focally positive for CD10 and negative for Tdt on immunohistochemistry. Mib1 index was nearly $100 \%$. In view of high Mib 1 index and strong Bcl 2 positivity and in the absence of disease elsewhere in the body on PET CT, a diagnosis of primary CNS B cell lymphoma, unclassifiable, with features intermediate between DLBCL and BL was given. Conclusion: Primary CNS B cell lymphoma, unclassifiable, with features intermediate between DLBCL and BL can also occur in young immuno-competent patients. Though rare, these lymphomas are also known to occur in the central nervous system.
\end{abstract}

Keywords: Brain, Burkitt lymphoma, Diffuse Large B cell Lymphoma, Headache, Immunohistochemistry, Vomiting.

\section{Introduction}

Primary central nervous system (CNS) lymphomas are very rare accounting to $<1 \%$ of NHL and $3-5 \%$ of all brain tumors [1]. Age of onset shows two peaks, childhood and advanced age (60-70 years) [2], with a slight male preponderance M:F:: 3:2 [1]. Histologically more than $90 \%$ of primary CNS lymphomas are high grade B cell lymphomas, remaining are $\mathrm{T}$ cell lymphomas and MALT (mucosa associated lymphoid tissue) lymphoma [3]. Primary CNS B cell lymphoma unclassifiable, with features intermediate between DLBCL and Burkitt's lymphoma in an immuno-competent young boy is very rare, with very few cases being described in the literature.

\section{Case Report}

A 14 year old boy presented with one week history of headache and vomiting. Routine hematological and biochemical investigations done were within normal limits. CT brain showed well defined moderate sized oval shaped mildly enhancing hyper-dense lesion in left intra-ventricular region with seeding in the temporal lobe and the suprasellar region, mild peri-lesional edema was also seen suggesting a neoplastic condition. Radiological diagnosis considered was primitive neuro-ectodermal tumor and lymphoma.

Tumor decompression was done and tumor was sent for squash cytology. Cytology revealed monomorphic population of medium 
sized lymphoid cells with non-cleaved nuclei and scant cytoplasm. Multi-nucleated cells, tingible body macrophages, lympho-glandular bodies and brisk mitosis were noted. A diagnosis of high grade non-Hodgkin's lymphoma was given on squash cytology. Tissue paraffin sections showed tumor cells with similar cytological features in sheets predominantly with a very conspicuous starry sky appearance. Angiocentricity was noted at focal areas. Immunohistochemistry was done which showed tumor cells diffusely positive for $\mathrm{CD} 20, \mathrm{BCl}$, $\mathrm{BCl} 2$, focally positive for $\mathrm{CD} 10$ and negative for Tdt. Mib1 index was nearly $100 \%$. In view of high Mib 1 index and strong $\mathrm{Bcl} 2$ positivity and in the absence of disease elsewhere in the body on PET CT, a diagnosis of primary CNS B cell lymphoma, unclassifiable, with features intermediate between diffuse large B cell lymphoma and Burkitts lymphoma was given. Molecular studies were advised.

\section{Discussion}

Since the brain is devoid of lymphatics/ resident lymphocytes, one of the hypotheses described for primary lymphoma in the central nervous system is that primary central nervous system lymphomas might represent the metastasis of an occult systemic lymphoma, eradicated by the intact immune system but escaping within the immune privileged CNS [1]. Clinical presentation of patients with lymphoma of central nervous system is broad, ranging from focal neurological deficits to symptoms due to increased intra-ventricular pressure which includes headache and vomiting.

MRI with gadolinium contrast is the imaging modality of choice, although CT scan can also be done. Solitary lesions are more common, involving the cerebral hemispheres more often, followed by thalamus, corpus callosum, periventricular region and cerebellum. Our case had multifocal lesions with large left ventricular mass with seeding in the left temporal lobe and suprasellar region. Other CNS lymphomas are primary

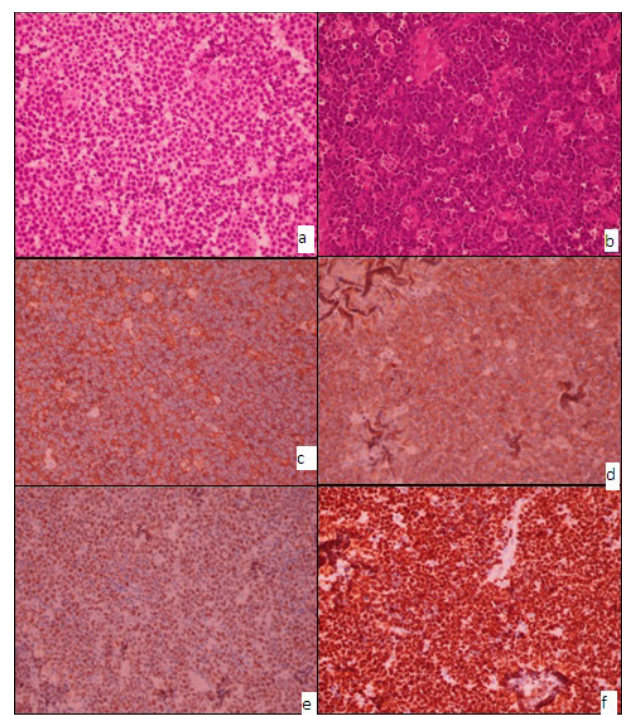

Fig.1(A): Squash cytology smear (Hematoxylin \& Eosin stain, original magnification x100), (B) Tissue paraffin block which shows starry sky appearance in a lymphoid background (Hematoxylin \& Eosin stain, original magnification x100). Immunohistochemistry images of tissue paraffin blocks (original magnification x100), (C) $\mathrm{CD} 20+$ (D) $\mathrm{Bcl} 2+$ (E) $\mathrm{Bcl6}^{+}$(F) Ki-67.

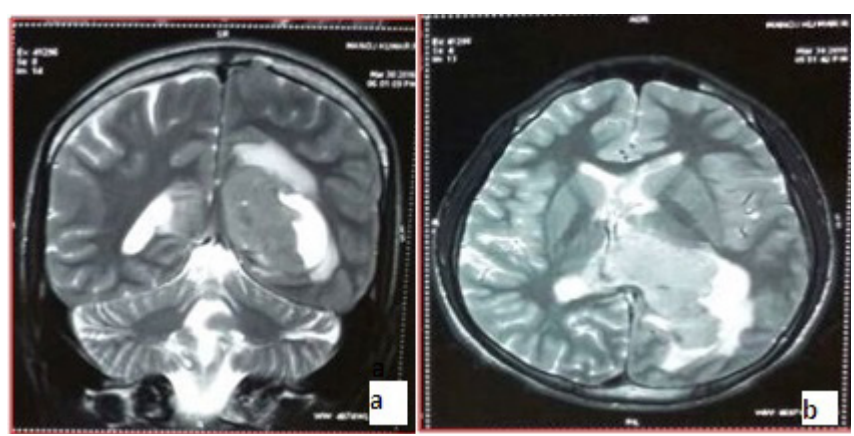

Fig.2: Post-contrast CT images of brain which shows oval shaped hyper-dense lesion in the left parietal region, corpus callosum with mild enhancement (as depicted in the arrow).

vitreo-retinal lymphoma constituting $<1 \%$ of NHL and are limited to the eye at the time of diagnosis. Meningeal lymphoma $(<5 \%)$, spinal cord lymphoma, spinal nerve and ganglia lymphomas are very rare [4].

Although CNS lymphoma have distinctive appearance on imaging, but none of the features can equivocally distinguish it from other brain lesions and hence-forth histological diagnosis of the 
primary central nervous system lymphoma is very essential for further management [5]. Histologically lymphomas have a distinctive morphological appearance and are characterized by dis-cohesive cells with relative monomorphic appearance [1]. The tumor cells of Burkitt's lymphoma are medium sized cells, nuclei have clumped to dispersed chromatin with multiple nucleoli. The tumor has high proliferation index and starry sky appearance is usually present. On immuno-histochemistry the tumor cells are positive for mature B cell antigens CD20, BC16, negative or weakly positive for BCL2 and negative for Tdt. Cases with atypical phenotype now placed under the category of B cell lymphoma unclassifiable, with features in between diffuse large B cell lymphoma and Burkitt's lymphoma (DLBCL/BL) are BCL2 positive and have all the features of Burkitt's lymphoma (BL) including IG: MYC translocation without BCL2 and BCL6 translocation [6]. Other high grade lymphomas considered in the differential diagnosis include diffuse large B cell lymphoma (DLBCL) and $\mathrm{B}$ cell lymphoma, unclassifiable, with features intermediate between DLBCL and BL (DLBCL/ $\mathrm{BL})$. DLBCL, morphologically cells are larger in size as compared to cells in $\mathrm{BL}$ and are positive for mature B cell antigens CD20, BCL6, BCL2 [5] and MUM1 [7]. Ki 67 is taken as critical criteria for the diagnosis of Burkitt's lymphoma. For distinguishing Burkitt's lymphoma and other aggressive B cell lymphoma cut off value of $\mathrm{Ki}$ 67 is 95 to $90 \%$. A proportion of DLBL show loss of BCL2 expression, and have Ki-67 index of more than $95 \%$. In addition to MYC translocation they also show other cytogenetic abnormality [8]. Cases that morphologically resemble Burkitt's lymphoma and are positive for BCL2 are placed under the category of double hit lymphoma when BCL2 and MYC translocations are present [9]. A systematic approach which includes morphology, immunohistochemistry and molecular studies is recommended for proper diagnosis of these aggressive B cell lymphoma due to significant overlapping between diffuse large B cell lymphoma, B cell lymphoma, unclassifiable, with features intermediate between diffuse large B cell lymphoma and Burkitt's lymphoma (DLBCL/BL) and Burkitt's lymphoma [8].

Other lymphomas considered in the differential diagnosis include low grade B cell lymphoma like Mantle cell lymphoma, $\mathrm{T}$ cell lymphoma and anaplastic large cell lymphoma [1]. Mantle cell lymphomas have slightly irregular nuclei and are positive for CD20, Cyclin D1and CD5 [10]. T cell lymphomas are also medium sized cells with minimal cytological atypia and pose a major diagnostic problem. T cell receptor gene rearrangement needs to be demonstrated [1].

Classical Burkitt's lymphoma is treated with high intensity multi-agent systemic chemotherapy regimens. Mis-diagnosing the disease as a less aggressive entity might result in sub-optimal or inadequate treatment (using R-CHOP- Rituximab regimen for DLBL). Regardless of MYC expression these cases which closely resemble Burkitt's lymphoma rather than a DLBCL should be treated as Burkitt's lymphoma. However, prognosis of primary central nervous system lymphomas is very poor [10].

\section{Conclusion}

Primary CNS lymphoma can also occur in young immuno-competent patients. Though rare, primary B cell lymphoma, unclassifiable, with features intermediate between DLBCL and Burkitt lymphoma can also occur in the central nervous system. Its molecular heterogeneity, aggressive behavior and poorer treatment outcome poses a major diagnostic and therapeutic challenge.

Contributors: MPP: manuscript writing, literature search; BP: manuscript editing, literature search. MPP will act as guarantor. Both authors approved the final version of the manuscript.

Funding: None; Competing interests: None stated. 


\section{References}

1. Gianini C, Dogan A, Saloma DR. CNS Lymphoma: A Practical Diagnostic Approach J Neurol. 2014;73:476494.

2. Sakurai H, Kei-Ji Sugimoto KJ, Shimada A, Imai H, Wakabayashi M, Sekiguchi Y, et al. Primary CNS CCND1/MYC- Positive Double-Hit B cell Lymphoma: A Case report and review of literature. J Clinical Oncol. 2015;33:79-83.

3. Jiang M, Zhu J, Guan YS, Li-quon Zon L. Primary Central nervous system Burkitt lymphoma with nonimmunoglobulin heavy chain translocation in right ventricle. Pediatric Hemato Oncol. 2011;28:454-458.

4. Del Rio MS, Rousseau A, Soussain C, Ricard D, HoangXuan K. Primary CNS Lymphoma in immunocompetent patients. The Oncologist. 2009;14:526-539.

5. Barrios L, Beckers M, Verhoef G, Dierickx D. Primary Central nervous system lymphoma: A case report. Belg J Hematol. 2016;7:118-122.
6. Haldorsena IS, Espeland A, Larsson EM. Central nervous system lymphoma characteristic findings on traditional and advanced imaging. American J Neuroradiol. 2011;32:984-992.

7. Rosewald A, Otti G. Burkitt lymphoma versus diffuse large B cell lymphoma. Annals of Oncology. 2008;19:6769.

8. Naresh KN, Ibrahim HAH, Lazzi S, Rince P, Onorati M, et al. Diagnosis of Burkitt lymphoma using an algorithmic approach - applicable in resource - poor and resource rich countries. British J Hematol. 2011;154:770-776.

9. Thomas DA, Brian SO, Manning Jr. JT, Romaguera J, et al. Burkitt lymphoma and atypical burkitt or Burkitt like lymphoma: Should be these be treated as different diseases. Curr Hematol Malig Rep. 2011;6:58-66.

10. Swerdlow SH, Campo E, Harris NL, Jaffe ES, Pileri SA, Stein H, et al. WHO Classification of tumours of Haematopoetic and Lymphoid tissue. 4th edition. Lyon: International Agency for Research on Cancer; 2008. pp.262-264. 